\title{
Primer reporte de Dendropsophus bogerti (Anura: Hylidae: Hylinae) en el departamento del Chocó, Colombia
}

\author{
First record of Dendropsophus bogerti (Anura: Hylidae: Hylinae) \\ in the department Choco, Colombia
}

\author{
Pablo Palacios-Rodríguez", Jhon Tailor Rengifo-Mosquera*, \\ Jonard David Echavarría-Renteria*
}

\begin{abstract}
Resumen
Se presenta un nuevo registro para una rana arbórea Dendropsophus bogerti en el oriente del departamento del Chocó, Colombia (05 $51^{\prime}$ ' 23,2"N, 76 11' 15.1"W: 1050 metros de elevación). D. bogerti se conocía en la región central-norte de la Cordillera Central, sobre los departamentos de Antioquia y Caldas. Este reporte se basó en una serie de individuos colectados en una zona de vida de un bosque pluvial montano bajo (bp-MB), con una fuerte presión agrícola y ganadera. Este registro se convierte en el reporte más occidental para esta especie en el país y el primero en el departamento del Chocó.
\end{abstract}

Palabras clave: Anuros, Distribución geográfica, Ranas de árbol.

\begin{abstract}
A new record for a tree frog Dendropsophus bogerti is presented in the east of the department of Chocó, Colombia $\left(05^{\circ} 51^{\prime} 23.2^{\prime \prime} \mathrm{N}, 76^{\circ} 11^{\prime} 15.1^{\prime \prime W}: 1050\right.$ elevation $D$. bogerti known in the northcentral region of the Central Andes, in the departments of Antioquia and Caldas. This report was based on a number of individuals collected in an area of life a montane rainforest (bp-MB), with strong pressure agriculture and livestock. This record becomes the westernmost report for this species in the country and the first in the department of Choco.
\end{abstract}

Keywords: Frogs, Geographic distribution, Tree frogs.

El género Dendropsophus Fitzinger, 1843 es el grupo de ranas más diverso de la familia Hylidae en Colombia con un total de 35 especies descritas hasta la fecha (Acosta-Galvis 2013). Dendropsophus bogerti (Cochran y Goin, 1970) (Figura 1), fue descrito basado en una hembra adulta, colectada por el hermano Nicéforo María en Medellín, departamento de Antioquia. Es una especie de tamaño moderado que se distribuye en la región central-norte de la cordillera central, sobre los departamentos de Antioquia y Caldas entre los 1256-2580 metros de elevación (Cochran y Goin 1970; Kaplan 1997, 1999; Acosta 2000; Rivera y Gutiérrez 2012).

La especie fue inicialmente descrita como Hyla bogerti y luego sinonimizada por Duellman (1974) como H. carnifex; pero no se presentó una justificación precisa para esta sinonimia. Luego, Duellman y Trueb (1983) proponen las similitudes en la coloración ventral y los patrones del flanco para justificar la sinonimia; aunque estuvieron basados únicamente en la revisión de especímenes preservados (H. carnifex para Ecuador y $H$. bogerti para Colombia). Subsecuentemente, Kaplan (1997) provee una revisión más completa de las poblaciones ecuatorianas y colombianas de $H$. carnifex y resucita a $H$. bogerti como una especie válida para las poblaciones colombianas, basado esto en la revisión de la coloración en vida, patrones de coloración de especímenes preservados, ecología de estos individuos y morfología del renacuajo. Posteriormente Faivovich et al. (2005),

* Grupo de investigación en Herpetología; Programa de Biología, Universidad Tecnológica del Chocó; Ciudadela Universitaria, Quibdó, Chocó, Colombia. e-mail: paparo92@gmail.com

Fecha recepción: Octubre 4, 2013 Fecha aprobación: Noviembre 27, $2013 \quad$ Editor asociado: Mantilla H. 


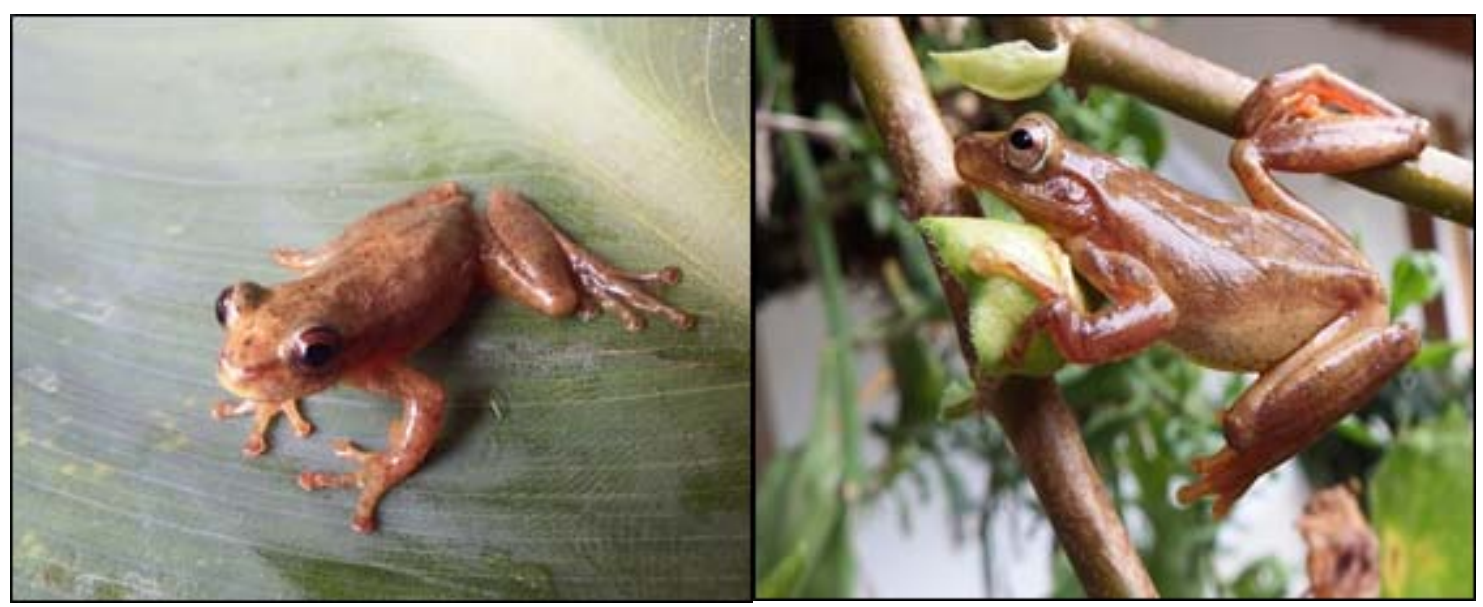

Figura 1. Individuos de Dendropsophus bogerti (izquierda: macho; derecha: hembra) colectados en el municipio de Carmen de Atrato, Chocó, Colombia.

presentando datos moleculares y cromosómicos, incluye dentro del género Dendrosophus, a todas las especies antes asignadas para el género Hyla, que se sabe o se sospecha presentan 30 cromosomas, ubicando a Hyla bogerti en el género Dendropsophus y a su vez como una especie perteneciente al grupo de D. columbianus (sensu Faivovich et al. 2005).

Los caracteres diagnósticos que permiten identificar a $D$. bogerti son: presencia de dos dientes vomerianos pequeños, lengua dos tercios de amplia con referencia a la apertura bucal; hocico corto, algo redondeado en vista dorsal, fosas nasales más laterales que superioriores, Canthus rostralis moderadamente bien definido; la región loreal es ligeramente cóncava y oblicua. Ojos pequeños y no tan prominentes con un diámetro igual a la distancia de la ventana de la nariz, presenta membrana palpebral no reticulada, distancia interorbital mayor que la anchura del párpado superior que es relativamente estrecha; tímpano bastante distinguible, de un tamaño más o menos de la mitad del diámetro del ojo y separado de este por una distancia aproximada igual a su diámetro, superficies dorsales un tanto lisas, sobre la región ventral; la garganta y el pecho son casi lisos, mientras que el vientre y la superficie de los muslos son finamente granulares.

El 18 de septiembre de 2013, como producto de trabajo de campo en la vía Quibdó-Medellín a la altura del kilómetro $10\left(05^{\circ} 51^{\prime} 23,2^{\prime}{ }^{\prime} \mathrm{N}, 76^{\circ} 11^{\prime} 15.1^{\prime \prime} \mathrm{W}\right.$ : $1050 \mathrm{msnm}$ ) y utilizando el método de búsqueda por encuentros visuales para anfibios (VES por sus siglas en inglés), se colectó una serie de ejemplares de tres machos (SVL: 25.6; SVL: 26.2; SVL: 25.2) y una hembra (SVL: 31.4) de D. bogerti (Figura 2). Los especímenes fueron encontrados vocalizando, durante una noche de lluvia entre las 21:50-22:03 horas, sobre un parche de vegetación que rodeaba un pequeño riachuelo. La zona corresponde a un bosque pluvial montano bajo (bp-MB) intervenido, dominado por actividades como la agricultura y la ganadería. Los ejemplares fueron depositados en la Colección Científica de Referencia Zoológica de la Universidad Tecnológica del Chocó (COLZOOCH-H 1376-79). De esta forma, nosotros presentamos aquí el primer registro para $D$. bogerti en el departamento del Chocó, el cual es el registro más occidental para esta especie en Colombia (Figura 3).

Según Kaplan (1997), D. bogerti es una especie muy adaptable en hábitats intervenidos y en pastizales formando grupos muy numerosos, por lo general con machos realizando cantos muy pronunciados. El hallazgo de esta especies en el departamento del Chocó, Colombia, representa un hecho de suma importancia en término de composición de este grupo faunístico en este departamento, lo cual aporta al objetivo de reconocer las especies de anfibios que ocurren en esta sección de las tierras bajas del pacífico colombiano.

\section{Agradecimientos}

Nuestros agradecimientos para la Vicerrectoría de Investigaciones y al grupo de investigación en Herpetología de la Universidad Tecnológica del Chocó. Agradecemos de manera muy especial al profesor 

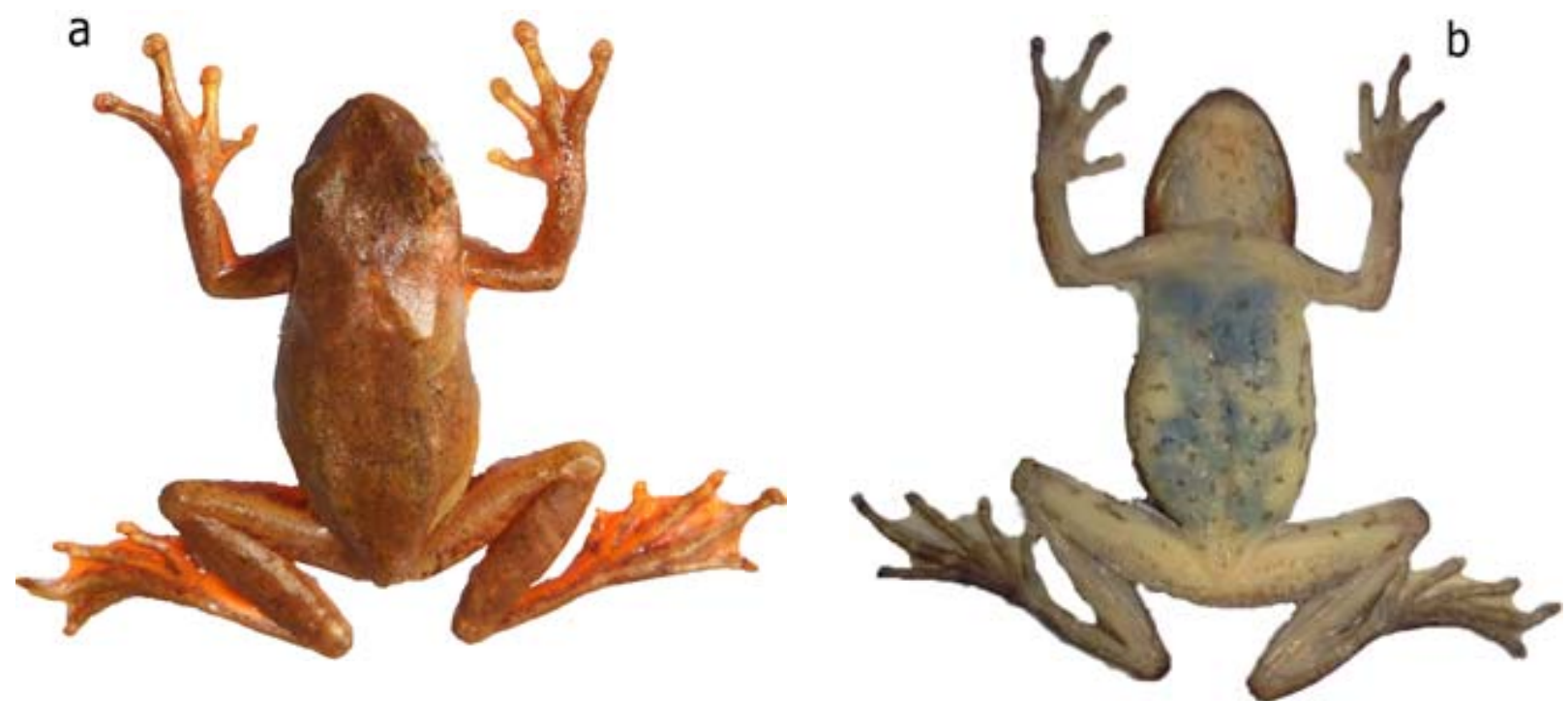

Figura 2. (a) vista dorsal, (b) vista ventral de una hembra de D. bogerti (COLZOOCH-H 1377), depositada en la colección Científica de Referencia Zoológica de la UTCH, Quibdó, Colombia.

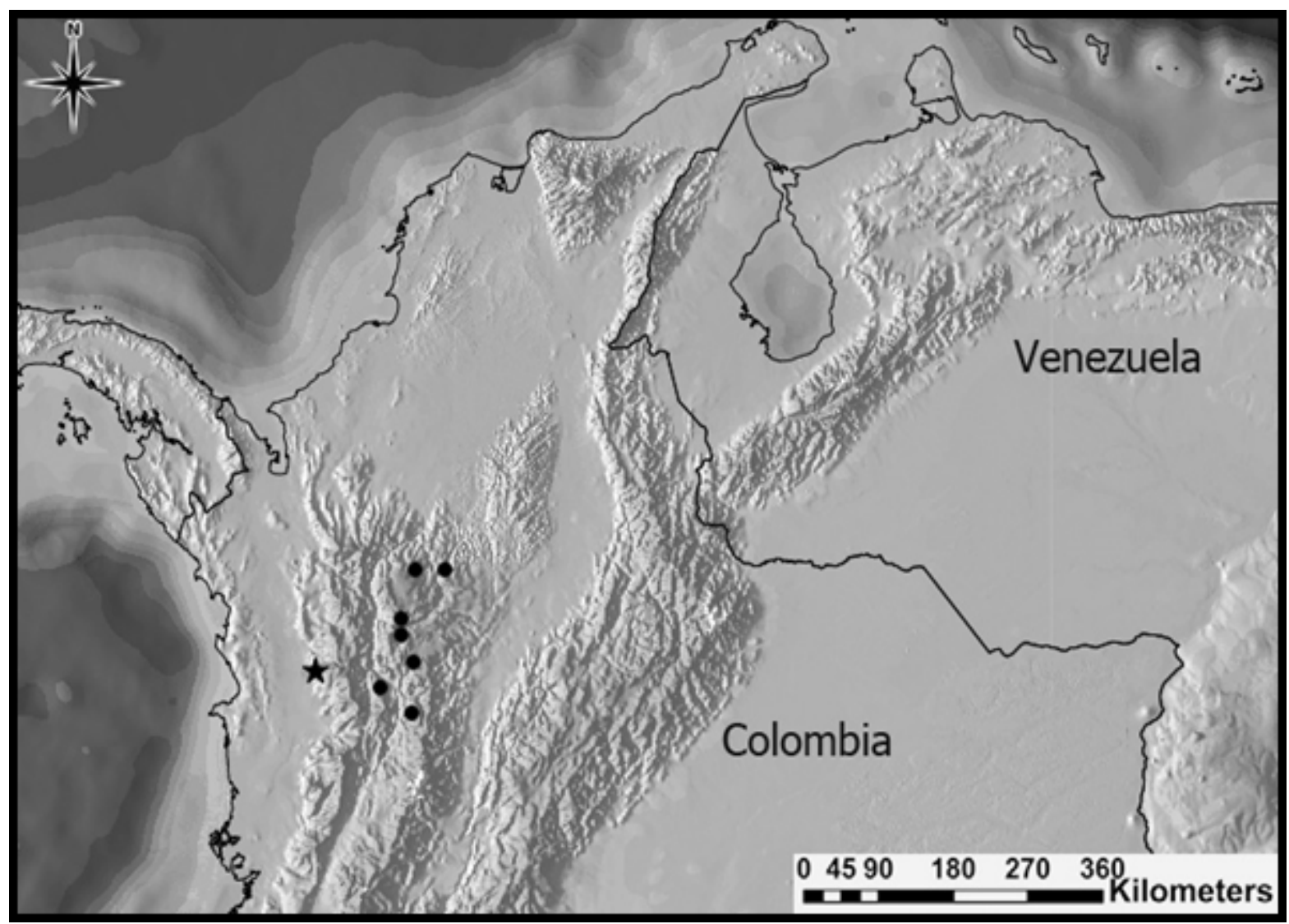

Figura 3. Distribución geográfica para $D$. bogerti en Colombia (estrella= nuevo reporte; círculos reportes de literatura).

Alex Jiménez Ortega por su gran apoyo. Este documento es dedicado al profesor Fabio García Cossio por la gran entrega que le brindó durante todos estos años al programa de Biología de la UTCH. 


\section{Literatura citada}

Acosta AR. 2000. Ranas, salamandras y caecilias (Tetrapoda: Amphibia) de Colombia. Biota Colomb. 289-319.

Acosta AR. 2013. Lista de los anfibios de Colombia. VV.02.2013.0. URL disponible en: www.batrachia.com Cochran DM, Goin CJ. 1970. Frogs of Colombia. Bull USA Nat Museum. 288: 1-655.

Crump ML, Scott NJ. 1994. Visual encounter surveys. In: Heyer W, Donnelley MA, McDiarmid RA, Hayec LC, Foster MC (eds) Measuring and monitoring biological diversity standard methods for amphibians. Washington, DC: Smithsonian Institution Press.

Duellman WE. 1974. A reassessment of the taxonomic status of some neotropical hylid frogs. Occas Pap Mus Nat Hist Univ Kansas. 27: 1-27.

Duellman WE. Trueb L. 1983. Frogs of the Hyla columbiana group: taxonomy and phylogenetic relationships. In: Rhodin AGJ, Miyata K. (Eds.). Advances in herpetology and evolutionary biology. Essays in Honor of Ernest E. Williams. Museum of Comparative Zoology, Harvard University [Cambridge, Massachusetts]. p. 33-51.

Faivovich J, Haddad CFB, García PCA, Frost DR, Campbell JA, Wheeler WC. 2005. Systematic review of the frog family Hylidae, with special reference to Hylinae: phylogenetic analysis and taxonomic revision. Bull Am Mus Nat Hist. 294: 1-240.

Kaplan M. 1997. On the status of Hyla bogerti Cochran and Goin. J Herpetol. 31: 536-41.

Kaplan M. 1999. On the phylogenetic relationships of Hyla praestans and the monophyly of the Hyla columbiana group: morphological observations of the larynx. Rev Colomb Cienc. 23 (Supl especial): 299-302.

Rivera M, Gutiérrez PDA. 2012. A new highland species of tree frog of the Dendropsophus columbianus group (Anura: Hylidae) from the Andes of Colombia. Zootaxa. 3486: 50-62. 